\title{
Using silkworms to search for lactic acid bacteria that contribute to infection prevention and improvement of hyperglycemia
}

\author{
Atsushi Miyashita ${ }^{1}$, Kazuhisa Sekimizu ${ }^{1,2, *}$ \\ ${ }^{1}$ Institute of Medical Mycology, Teikyo University, Tokyo, Japan; \\ ${ }^{2}$ Genome Pharmaceuticals Institute Co., Ltd., Tokyo, Japan.
}

SUMMARY Bombyx mori, the silkworm, has biological functions in common with mammals, including humans. Since the molecular design of silkworm's innate immune system is analogous to that of mammals, understanding the silkworm's innate immunity is expected to contribute to the control of infection in humans. It is also possible to use silkworms to explore foodstuffs that activate innate immunity. Lactic acid bacteria have long been used in the production of fermented foods, and in recent years, their use as supplements has been attracting attention. Using silkworms, which are laboratory animals, functional lactic acid bacteria can be explored and isolated at low cost. Fermented foods produced by this method are expected to contribute to the maintenance of human health. In addition to the immune system, humans and silkworms share a common mechanism for maintaining blood glucose homeostasis, and it is possible to construct a pathological model of diabetes and search for therapeutic substances using silkworms. Taken together, we propose that the silkworm is useful for assessing the functions of lactic acid bacterial for health purposes.

Keywords silkworm, functional foods, immune priming, diabetes

\section{Introduction}

With the outbreak of the new coronavirus infection, functional foods that enhance immunity have been attracting attention. In addition, in Japan's super-aging society, there is growing interest in the prevention of lifestyle-related diseases such as diabetes (1). Lactic acid bacteria is one of the best food materials to meet these needs and have been used in the production of fermented foods such as pickles, yogurt, and breads since ancient times $(2,3)$. Recently, the usefulness of the lactic acid bacteria for health maintenance has been emphasized. Maintaining people's health by consuming natural and functional foods that utilize lactic acid bacteria will provide a new approach to combating these diseases, which until now have relied on antibiotics and diabetic drugs. A variety of lactic acid bacteria are sold as functional foods for health promotion. However, there are not many of these products that have solid evidence (4-6). In general, foods are often evaluated for their health using mammals such as mice. However, it has been pointed out that using mice is not only costly but also ethically problematic in terms of animal welfare (7). Therefore, it is becoming increasingly difficult to obtain evidence from animal experiments using mice. Furthermore, in order to obtain evidence for health foods, it is ultimately necessary to conduct tests using humans. However, this requires huge costs (\$3000-5000 per human subject (8)), and for this reason, health foods are currently being sold with little scientific evidence.

In an attempt to solve this problem, we have made use of silkworms. The silkworms have been used for sericulture since ancient times and have produced silk as a domesticated insect species. Japan has been at the forefront of the world in terms of silkworm breeding methods and strain maintenance. Recently, the production of genetically modified silkworms has become possible, and the silkworm has acquired the status of an experimental material for cutting-edge biology (9). However, since the silkworm 'looks' very different from humans, many people wonder whether the results of experiments on silkworms can be applied to humans. Let us consider what functions humans have that silkworms do not. The silkworm feeds on mulberry leaves. The silkworm recognizes mulberry leaves as food, and this is due to the function of the silkworm's brain. The mulberry leaves are digested in the digestive tract, and the nutrients are absorbed into the bloodstream. The silkworm has the muscles and nervous system necessary for feeding behavior. Silkworms also have blood, but not red-colored because it lacks the red blood 
cells. The silkworm's blood contains immune cells that correspond to human white blood cells. Invertebrates, including the silkworm, have no genes encoding antibodies and they protect themselves from pathogens using an antibody-independent immune mechanism called innate immunity (10-13). Although it is true that the silkworm does not have some aspects of human body functions (e.g., it lacks bones so that it may not be the best animal to create an osteoporosis model), most of the basic biological functions are shared by both silkworms and humans. Therefore, in principle, it is possible to create models of many diseases that humans suffer from using silkworms. We have mainly studied infectious disease models in silkworms, where we have found that most of the bacteria and fungi that infect mammals can infect and kill silkworms $(14,15)$. We have also reported that antibiotics used as therapeutic agents in human clinical practice were effective in the silkworm infection model (16), and that the $\mathrm{ED}_{50}$ value, an index of therapeutic efficacy, was consistent between silkworms and mammals (15). We conducted a search for antibiotics against Staphylococcus aureus using this silkworm infection model as a screening tool for therapeutic efficacy, and succeeded in discovering Lysocin E, an anti-MRSA infection treatment, from extracts of soil bacteria (17). Animal studies on Lysosin E were conducted (unpublished), and it is now at the stage of planning human trials.

\section{Innate immunity activation test using the silkworm muscle contraction system}

As mentioned above, invertebrates rely on innate immunity for pathogen defense. This make a merit for the silkworm to be an innate immunity model, because, in mammals, it is not always easy to quantitatively measure innate immune activation at the individual level due the intricated cross-talks and interactions between the innate immune system and the adaptive immune system. Although Drosophila (fruit flies) and Caenorhabditis elegans (nematodes) have been used in biological research, the body size of the silkworm (they are much larger than fruit flies and nematodes) makes it easier to collect a good amount of blood sample by cutting its legs with scissors and to inject a certain volume of sample solution into the blood stream using a tuberculin syringe with needle. In addition, organs such as the intestinal tract can be removed to conduct experiments such as drug permeability test (15).

We reported that the silkworm's immune system is activated by the cell wall components of bacteria and fungi, resulting in contraction of silkworm muscles (18). This is a phenomenon in which immunocompetent cells in the silkworm's blood (often called hemolymph) recognize pattern molecules via innate immune receptors and release reactive oxygen species that lead to activation of paralytic peptides (BmPP; an insect cytokine), and then muscle contraction as a pharmacological effect of BmPP. Using this unique function, it is possible to quantitatively evaluate innate immunity activators in foods using the silkworm muscle contraction assay system.

The advantage of the silkworm muscle contraction as an indicator of innate immune activation is that it does not require costly facilities such as fancy fluorescent microscopes or mass spectrograms. It is worth noting here that the muscle contraction of the silkworm is not affected by lipopolysaccharides (LPS), which is always a problem in cell culture-based in vitro immune activation tests. LPS is a component of the outer wall of Gramnegative bacteria such as Escherichia coli, but since it acts on mammalian immune cells at extremely low concentrations, it is known to be easily contaminated by environmental contaminants such as tap water, which frequently affect the quantification of immunoreactive substances. It is a huge technical merit that the muscle contraction system can evaluate the activation of innate immunity without being affected by LPS. Using the silkworm muscle contraction system, we have found that extracts of green tea and broccoli have high immunoreactivity. Also, some lactic acid bacteria show very high immunoreactivity in this system. When various lactic acid bacteria were collected and tested, they show a wide range of activity from less than 1 units/mg to 105 units/mg (Table 1). It is also possible to identify subspecies of lactic acid bacteria that have outstanding immunoreactivities. We have identified a subspecies of Lactococcus lactis, 11/19-B1, using this system (19).

\section{Primed immune responses found in the silkworm}

When Pseudomonas aeruginosa is injected into the silkworm after pre-administration of an immune

Table 1. Immunoreactivity (silkworm muscle contraction activity) of different lactic acid bacteria.

\begin{tabular}{llc}
\hline No. & Phylogenetic Identity & Activity (U/mg) \\
\hline 1 & Lactococcus lactis & 105 \\
2 & ND (Not Determined) & 77 \\
3 & Lactococcus lactis & 45 \\
4 & Streptococcus thermophilus & 43 \\
5 & Lactococcus lactis & 33 \\
6 & Lactobacillus bulgalicus & 29 \\
7 & ND & 28 \\
8 & Lactobacillus bulgalicus & 24 \\
9 & ND & 23 \\
10 & Lactobacillus casei & 18 \\
11 & Lactococcus lactis & 17 \\
12 & ND & 7.1 \\
13 & ND & 2.2 \\
14 & ND & 2 \\
15 & Enterococcus casseliflavus & 0.83 \\
16 & ND & 0.45 \\
17 & ND & $<0.30$ \\
\hline
\end{tabular}

A list of lactic acid bacteria is shown in the table with corresponding muscle contraction activity. Data from our previous work (19). 
activator (immune primer), the silkworm may become resistant to $P$. aeruginosa infection. This function is called the primed immune responses in silkworms (12,20). P. aeruginosa, a Gram-negative bacterium, is a causative agent of opportunistic infections in humans and is naturally resistant to many antibiotics. More recently, nosocomial infections caused by multidrug-resistant $P$. aeruginosa (MDRP) have become a problem. We have found that several lactic acid bacteria are effective in this system. It is noteworthy that lactic acid bacteria can be added in the diet of silkworm, i.e., oral administration shows immune-priming effects and protects silkworms from infection. In our past experience of searching for antibiotics, the probability of finding a drug that works against Gram-negative bacteria is extremely low. Therefore, it was a great surprise when we discovered this orally effective measure against $P$. aeruginosa infection. We are currently working on purification of the active substance, identification of its chemical structure, and the molecular mechanism of its action.

\section{Evaluation of hypoglycemic substances using silkworms}

For the uninitiated, it may come as a surprise that silkworms have blood and blood glucose levels. In the long history of sericulture, silkworms have probably never been fed sweets. Yet, in fact, when the silkworm is fed sugar, the blood glucose level rises and the weight gain stops, where glycation of blood proteins similar to that in human clinical practice is found. Insulin is a drug prescribed for diabetic patients, but even in silkworms whose blood glucose levels are elevated by the administration of sucrose, injecting human recombinant insulin lowers the blood glucose levels (21). Insulin also cancels the growth inhibition caused by the elevated blood glucose levels (21). It is also known that prolonged administration of glucose to mammals for more than a year can lead to type 2 diabetes, a condition in which insulin is ineffective. In the case of silkworms, continuous administration of glucose for only one day results in insulin resistance. Moreover, type 2 diabetes drugs such as metformin are effective in silkworms. These results suggest that the mechanism of blood glucose maintenance in the silkworm is analogous to that in mammals. In fact, the phosphorylation of Akt protein in silkworm fat body cells (a counterpart of human adipocytes) is increased by insulin and decreased by the inhibitor wortmannin, showing the similarity between silkworm and mammals at the molecular level (21).

We used this silkworm model of diabetes to search for ingredients in foodstuffs that exhibit hypoglycemic effects. Enterococcus faecalis is a lactic acid bacterium that causes human diseases as enterococci, but nonpathogenic variants are known and used in the production of various fermented foods. We found a sub-strain named YM0831 that was capable of lowering blood glucose levels in silkworms (22). We also confirmed that this bacterium has no pathogenic gene and is safe as food. The bacterium was found to suppress glucose uptake in vitro by Caco-2 cells derived from the human intestinal tract (22). This may be the mechanism of the hypoglycemic effect of this bacterium in the silkworm. We also conducted a blood glucose lowering test using healthy human volunteers. The results showed that the sucrose-induced increase in blood glucose level was suppressed by prior oral intake of the live bacteria (22). This finding endorses the usefulness of silkworm model for the search of human hyperglycemia drugs.

\section{Future development}

Lactic acid bacteria have been used in various fermented foods as non-pathogenic bacteria. We would propose a novel approach to evaluate the immune-activating and blood glucose-lowering effects of lactic acid bacteria using silkworm models, providing promising paths towards human trials on lactic acid bacteria if showing outstanding effects. This new approach will avoid the huge cost of conducting unpromising human trials and the ethical problem of mammalian sacrifice. The approach can be applied to: development of pet foods to which functional lactic acid bacteria are added, feed for farmed fish and livestock, and supplements for humans. Food products fermented with functional lactic acid bacteria can be expected to have a variety of health benefits and are the potent target of commercialization.

\section{Funding: None.}

Conflict of Interest: The authors have no conflicts of interest to disclose.

\section{References}

1. Morimoto A, Nishimura R, Tajima N. Trends in the epidemiology of patients with diabetes in Japan. Japan Med Assoc J. 2010; 53:36-40.

2. Jana T, Pathak S. Biotechnology for human welfare: past and road ahead. Biotech Nat. 2018; 13-33.

3. Shevchenko A, Yang Y, Knaust A, Thomas H, Jiang H, Lu E, Wang C, Shevchenko A. Proteomics identifies the composition and manufacturing recipe of the 2500-year old sourdough bread from Subeixi cemetery in China. J Proteomics. 2014; 105:363-371.

4. McNaught CE, MacFie J. Probiotics in clinical practice: a critical review of the evidence. Nutr Res. 2001; 21:343353.

5. Pham M, Lemberg DA, Day AS. Probiotics: sorting the evidence from the myths. Med J Aust. 2008; 188:304-308.

6. Ehrlich SD. Probiotics - little evidence for a link to obesity. Nat Rev Microbiol. 2009; 7:901.

7. Miyashita A, Iyoda S, Ishii K, Hamamoto H, Sekimizu $\mathrm{K}$, Kaito C. Lipopolysaccharide O-antigen of enterohemorrhagic Escherichia coli O157: H7 is required 
for killing both insects and mammals. FEMS microbiol Lett. 2012; 333:59-68.

8. Frestedt J. Similarities and difference between clinical trials for foods and drugs. Austin J Nutr Food Sci. 2017; 5:1086.

9. Daimon T, Kiuchi T, Takasu Y. Recent progress in genome engineering techniques in the silkworm, Bombyx mori. Dev Growth Differ. 2014; 56:14-25.

10. Miyashita A, Lee TYM, Adamo SA. High-stakes decisionmaking by female crickets (Gryllus texensis): When to trade in wing muscles for eggs. Physiol Biochem Zool. 2020; 93:450-465.

11. Miyashita A, Lee TYM, McMillan LE, Easy R, Adamo SA. Immunity for nothing and the eggs for free: Apparent lack of both physiological trade-offs and terminal reproductive investment in female crickets (Gryllus texensis). PLoS One. 2019; 14:e0209957.

12. Miyashita A, Kizaki H, Kawasaki K, Sekimizu K, Kaito C. Primed immune responses to gram-negative peptidoglycans confer infection resistance in silkworms. J Biol Chem. 2014; 289:14412-14421.

13. Miyashita A, Adamo SA. Stayin'alive: endocrinological stress responses in insects. In: Advances in invertebrate (neuro) endocrinology. Apple Academic Press, 2020; pp. 283-323.

14. Kaito C, Kurokawa K, Matsumoto Y, Terao Y, Kawabata S, Hamada S, Sekimizu K. Silkworm pathogenic bacteria infection model for identification of novel virulence genes. Mol Microbiol. 2005; 56:934-944.

15. Hamamoto H, Kurokawa K, Kaito C, Kamura K, Manitra Razanajatovo I, Kusuhara H, Santa T, Sekimizu K. Quantitative evaluation of the therapeutic effects of antibiotics using silkworms infected with human pathogenic microorganisms. Antimicrob Agents Chemother. 2004; 48:774-779.
16. Kaito C, Akimitsu N, Watanabe H, Sekimizu K. Silkworm larvae as an animal model of bacterial infection pathogenic to humans. Microb Pathog. 2002; 32:183-190.

17. Hamamoto H, Urai M, Ishii $\mathrm{K}$, et al. Lysocin $\mathrm{E}$ is a new antibiotic that targets menaquinone in the bacterial membrane. Nat Chem Biol. 2015; 11:127-133.

18. Ishii K, Hamamoto H, Kamimura M, Sekimizu K. Activation of the silkworm cytokine by bacterial and fungal cell wall components via a reactive oxygen speciestriggered mechanism. J Biol Chem. 2008; 283:2185-2191.

19. Nishida S, Ono Y, Sekimizu K. Lactic acid bacteria activating innate immunity improve survival in bacterial infection model of silkworm. Drug Discov Ther. 2016; 10:49-56.

20. Miyashita A, Takahashi S, Ishii K, Sekimizu K, Kaito C. Primed immune responses triggered by ingested bacteria lead to systemic infection tolerance in silkworms. PLoS One. 2015; 10:e130486.

21. Matsumoto Y, Sumiya E, Sugita T, Sekimizu K. An invertebrate hyperglycemic model for the identification of anti-diabetic drugs. PLoS One. 2011; 6:e18292.

22. Matsumoto Y, Ishii M, Hasegawa S, Sekimizu K. Enterococcus faecalis YM0831 suppresses sucroseinduced hyperglycemia in a silkworm model and in humans. Commun Biol. 2019; 2:157.

Received February 23, 2021; Accepted March 9, 2021.

*Address correspondence to:

Kazuhisa Sekimizu, Institute of Medical Mycology, Teikyo University. 359 Otsuka, Hachioji, Tokyo 192-0352, Japan.

E-mail: sekimizu@main.teikyo-u.ac.jp

Released online in J-STAGE as advance publication March 19, 2021. 